\section{Varied reward sequence and resistance to extinction in the straight alley'} DALE W. LEONARD, University of
Rochester, Rochester, N.Y, 14627

Five groups of rats were extinguished in a straight alley after training on one of the following reward sequences: $S M, M M, L M$, $M S, M L$. Groups $S M, M M$, and $L M$ were used to test the hypothesis that smaller rewards on the first trial on a two-trial varied reward sequence increase resistance to extinction (Rn). Groups $M S, M M$, and $M L$ tested the hypothesis that larger rewards on Trial 2 increase $R n$ in such groups. The results, in general, supported these predictions.

According to sequential theory, varied reward groups exhibit increased resistance to extinction $(\mathrm{Rn})$ compared to constant reward groups because they experience trial sequences in which large reward (L) follows small reward (S). Thus Leonard (1969) showed that a group given SL sequences alone was more resistant than two constant-reward groups and a second varied-reward group trained with LS sequences only.

The theoretical interpretation of such two-trial reward sequences is that (a) the first reward event provides a stimulus aftereffect $^{2}$ more or less similar to the SN extinction aftereffect, and (b) the second reward event conditions that aftereffect strongly or weakly depending on the size of the Trial 2 reward. Thus maximum $R n$ can be generated by keeping Trial 1 reward small

The present experimental design provides a more complete test of these two principles. Five groups of rats were given two trials each day in one of the following sequences: $S M$, MM, LM, MS, ML (M denotes medium reward). Thus, a comparison of Groups SM, MM, and LM evaluates the effect of holding Trial 2 reward constant and varying rew ard and making Trial 2 reward large. on Trial 1. A comparison of Groups MS, $M M$, and ML evaluates the effects of holding Trial 1 reward constant and varying Trial 2 reward. The predictions from sequential theory are shown in Fig. 1 where the abscissa indicates the stimulus being conditioned and the ordinate represents the strength of conditioning. Within each comparison the groups with the greater amount of generalized associative strength at SN will theoretically exhibit greater $\mathrm{Rn}$.

\section{SUBJECTS}

The Ss were 60 male Holtzman rats, approximately 100 days old.

\section{APPARATUS}

A $6 \times 4 \times 56$ in. alley, constructed of plywood and painted light gray, was used. A steel guillotine door, located 12 in. in front of a brass food cup, separated the goal area from the rest of the alley. Timing was accomplished by the use of two photocells, the last located 1 in. in front of the food cup. The photocells were $48 \mathrm{in}$. apart and were connected to a Standard Electric .01 -sec timer. Noyes .045-g sucrose pellets were used as reward.

\section{PROCEDURE}

Upon arrival Ss were fed and watered ad lib for 3 days. then placed on a 14-g-per-day food-deprivation schedule. Deprivation con. tinued for 10 days prior to experimental training, during which time Ss were handled individually and in small groups. Ss also received at least six goal-placement trials in which they were placed directly in the enclosed goal area and allowed to consume four pellets. All Ss learned to eat the pellets during the placement trials.

The Ss were then randomly divided into five groups, and trained for 10 days at two trials per day under one of the following schedules: SM, MM, LM, MS, ML, where S, $M$, and $L$ denote a small ( 1 pellet), medium (4 pellets), or a large (16 pellets) reward.
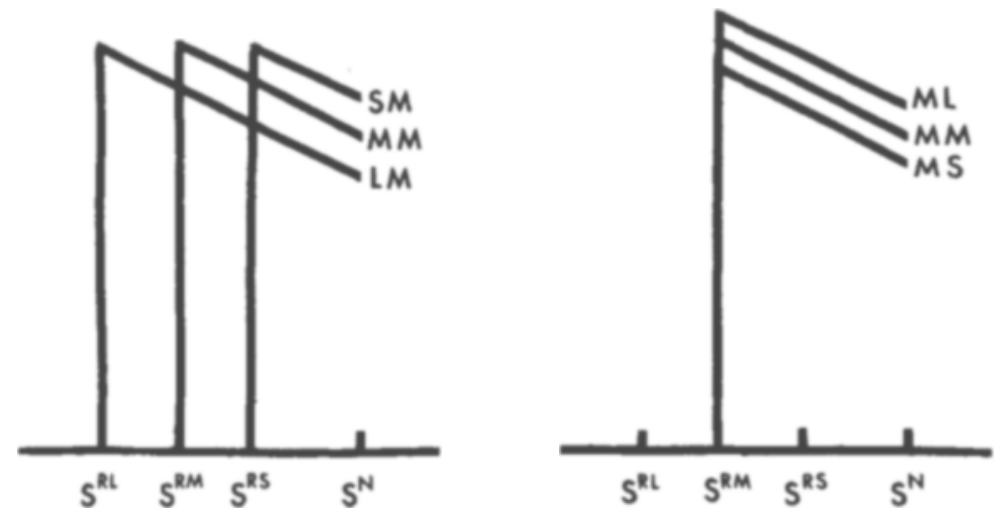

Thus, for example, Group SM received a single pellet on Trial 1 followed by 4 pellets on Trial 2 of each of its 10 training days. Ss were run in squads of five, each squad containing a single $\mathrm{S}$ from each experimental group, so that the intertrial interval was the time needed to run the other four Ss in the squad (approximately 3-4 min).

On the day following the completion of training, all Ss were given nine extinction trials. On extinction trials Ss were confined in the unbaited goal area for $30 \mathrm{sec}$. Ss were again run in squad rotation during extinction. Experiment 1 was completed in two identical replications, each employing 30 Ss. In both replications training was staggered so that only $10 \mathrm{Ss}$ began the extinction phase on a given day.

\section{RESULTS}

Running times in the 4-ft alley section were first converted to speeds $(\mathrm{ft} / \mathrm{sec}$ ) for purposes of statistical analysis. Each S's average speed on the final day of training was used to determine whether or not the groups differed prior to extinction. A one-way analysis of variance revealed no significant differences due to acquisition treatment $(F=1.86, \mathrm{df}=4 / 54, \mathrm{p}>.10)$.

The running speed of each of the five groups on the nine extinction trials are shown in Fig. 2. The left panel shows all groups given equal (medium) reward on Trial 2, and the right panel shows all groups with equal reward on Trial 1 of each training day. Group MM is plotted in both panels for comparison purposes.

Extinction speeds on the last eight extinction trials (excluding Trial "O") were grouped into four blocks of two trials whereupon a three-way analysis of variance with experimental groups (G), replications $(R)$, and trial blocks (T) as variance components was performed.

The fact that the experiment was conducted in two separate replications did not appear to have influenced the results since neither the $R$ component $(F=1.77$, $\mathrm{df}=1 / 50, \quad \mathrm{p}>.10)$ nor the $R$ by $\mathrm{G}$ interaction $(F=1.45$, df $=4 / 50, p>.10)$ reached significance. The $R$ by $T$ component was significant, however $(F=4.53$, $\mathrm{df}=3 / 150, \mathrm{p}<.01)$, indicating somewhat faster extinction in the second replication. Trial blocks was also a highly significant component $(F=276.16, \quad d f=3 / 150$, $\mathrm{p}<.01$ ), as expected.

The effect of the different training

Fig. 1. Theoretical predictions of group performance by the sequential hypothesis. The symbols SRL, SRM, and SRS represent the stimulus aftereffects of a large, medium, or small reward event, and the amount of habit generalization to the extinction aftereffect, $S N$, is shown. 
sequences on extinction performance was shown by the significant groups component $(F=19.04$, df $=4 / 50, p<.01)$ and the $G$ by $T$ interaction $(F=6.00$, df $=12 / 150$, $\mathrm{p}<.01)$. $T$ tests between individual pairs of groups based on each S's average speed throughout extinction were also performed. It was found that Group SM was more resistant to extinction than any other group ( $<<.001)$; Group ML was more resistant than $M M(p<.01)$ and $L M(p<.001)$ but was borderline with respect to MS $(.05<\mathrm{p}<.10)$. None of the other differences tested was significant.

\section{DISCUSSION}

In general, the ordering of the groups in extinction was in close agreement with predictions from sequential theory. For the three groups which received equal rewards on Trial 2 of each training day (SM, MM, LM), small reward on Trial 1 tended to increase $R n$ as predicted, although the difference between MM and LM was not significant by the $t$ test. This finding agrees with the sequential interpretation that different aftereffects are generated by the Trial 1 reward amounts, and conditioned on Trial 2 by (medium) reward, and produce resistance to extinction inversely proportional to the Trial 1 amount.

When the same aftereffect (SRM) was generated on Trial 1 in Groups MS, MM, and ML, but conditioned by varying amounts of reward on Trial 2, the prediction that greater $\mathrm{Rn}$ should obtain with larger Trial 2 reward was only partially verified. Group ML was most resistant, as predicted, but Groups MM and MS did not differ. ${ }^{3}$ The effect of the magnitude variable appeared to be much less when reward was varied on Trial 2 rather than on Trial 1.

The large differences in extinction performance between groups which received the same total amount of reward each day but differed in the reward sequence (ML vs LM; SM vs MS) were expected by the hypothesis and verify the previous results of Leonard (1969) for groups trained SL and LS. The former groups in each comparison have the double advantage in that a smaller Trial 1 aftereffect is conditioned more strongly by a larger Trial 2 reward.

\section{REFERENCES}

CAPALDI, E. J. A sequential hypothesis of instrumental learning. In $\mathrm{K}$. W. Spence and J.T. Spence (Eds.), The psychology of learning and motivation: Advances in research and theory.

Vol. 1. New York: Academic Press, 1967. Pp. 67-156.

CAPALDI, E. J., \& SPIVEY, J. E. Stimulus consequences of reinforcement and nonrein forcement: Stimulus traces or memory. Psy chonomic Science, 1964, 1,403-404.

LEONARD, D. W. Amount and sequence of reward in partial and continuous reinforcement. Journal of Comparative \& Physiological Psychology, 1969, 67, 204-211.
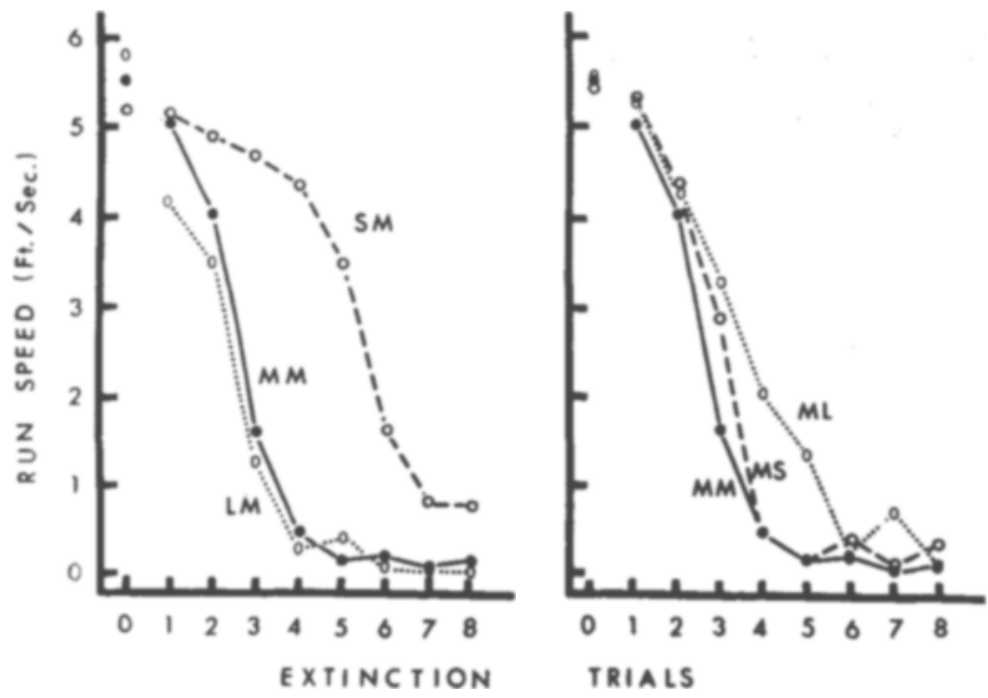

Fig. 2. Extinction performance of the five experimental groups on the nine extinction trials. Group MM is plotted twice for comparison purposes.

SHEFFIELD, V. F. Extinction as a function of partial reinforcement and distribution of practice. Joumal of Experimental Psy chology, $1949,39,511-526$.

\section{NOTES}

1. This investigation was supported in part by Research Grants MH-15704-01 and MH-10825-03 from the National Institute of Mental Health, and by the Center for Naval Analysis of the University of Rochester, Contract N00014-68A-0091. Such support does not imply endorsement of the content by the Navy.

2. The term "aftereffect" is used only for convenience, and is not meant to imply equivalence to the short-term trace relating to food in the teeth, etc., as originally conceived by Sheffield (1949). Capaldi feels the stimulus involved is long-term, perhaps more akin to a memory than to a simple trace (e.g., Capaldi \& Spivey, 1966), but its precise status is still in doubt.

3. A third replication using standard Noyes pellets and involving only the three groups in question resulted in the predicted extinction order, $\mathrm{ML}>\mathrm{MM}>\mathrm{MS}$; however, the difference between MM and MS again did not reach accepted levels of significance. Group ML was significantly more resistant than either of the other two groups as predicted. These Ss were run by Linda Leighton and Betsey Bensen.

\section{Performance under massed or spaced extinction following different sequences of varied reward training}

\section{DALE W. LEONARD, RICHARD ALBIN, and MARK LEBOWITZ, University of Rochester, Rochester, N.Y. 14627}

Rats were run in a straight alley to test an unusual prediction by sequential theory to the effect that a varied reward group trained with an SL reward sequence would tend to be more resistant than an $L S$ group under massed extinction, but less resistant under spaced extinction. A significant interaction between sequence and type of extinction was obtained. Also, a random varied reward group exhibited the greatest resistance under either extinction condition.

Leonard (1969) has reported that a varied-reward group given two daily training trials each day in a small-large reward sequence (SL) was more resistant to extinction than a group trained LS each day. This result was interpreted within the framework of Capaldi's (1967) sequential learning theory. 